\title{
User Services in BPAFrame, a Framework for Workflow-Management-Systems
}

\author{
${ }^{b}$ Mittasch, Ch.; ${ }^{b}$ Irmscher, K.; ${ }^{a}$ Ziegert,Th.; ${ }^{a}$ Lodderstedt, T.; ${ }^{a}$ Müller, \\ St.; ${ }^{a}$ Sommerfeld, $K$. \\ ${ }^{a}$ Dresden University of Technology, Dept. of Computer Science \\ D-01062 Dresden, Germany, ziegert@ibdr.inf.tu-dresden.de \\ ${ }^{b}$ Freiberg University of Technology, Dept. of Computer Science \\ D-09596 Freiberg, Germany, chris@informatik.tu-freiberg.de
}

\begin{abstract}
The paper describes the design and prototyping of BPAFrame (Framework for Business Process Automation) and shows its application in environmental information tasks. The design goals are outlined including the main factors: required user services, decentralized control, and an application dependent discussion of usable middleware-platforms. The main focus of this contribution is to discuss the requirements, components and realization of a user service framework for workflow management systems. Then the implemented prototype is introduced, and an application scenario is shown.
\end{abstract}

\section{Keywords}

CSCW, Workflow-Management, Distributed Computing, Distributed Platforms

\section{INTRODUCTION}

Workflows are becoming more and more relevant in office applications for both: business tasks i.e. ordering, booking and accounting; and for the administration management of different application areas. Workflow-Management-Systems (WfMS) support their assistance and partially their automation (Joosten, 1995). The main goals of WfMS are the control of workflows, the coordination of users, the control of appropriate data and a stepwise approach to an IT-representation of all business data within an organization. Thus, fluid internal processing and transparency are short-term goals of the use of WfMS. Furthermore, an iteration of a proper representation of all business objects (i.e. users, services, documents,.. and workflows) is becoming necessary for business process reengineering and fast and reliable reaction to changes in business tasks. 
Earlier research approaches addressed some of the above mentioned problems; examples are Action Paths, EuroCoop, and IPSO (Mittasch, Schill, 1996). These systems resulted in basic prototypes but hardly provided language and tool support. More recently, several workflow management products emerged; some examples are COSA, FlowMark, WorkParty and Prominand (Mittasch, Schill, 1996). These systems support comfortable workflow specification techniques and enable the integration of existing standard applications such as text processing or spreadsheets. However, they are typically working as extensions of centralized databases for implementing workflow management at runtime. This leads to wellknown problems of single points of failure, to potential performance bottlenecks, and also to a lack of scaleability, decentralization, dynamic reconfigurability and adaptability to different platforms and application areas.

Furthermore, we used the experience with our object-oriented WfMS CodAlf on top of DCE/DC++ (Distributed Computing Environment with Distributed C++) (Mittasch, 1996), (Schill, 1996), which allowed us to analyze the requirements of WfMS in more detail.

We have considered therefore that new WfMS have to fulfill the following requirements:

- to use open, distributed platforms, that allows the adoption of different hard- and software environments,

- to integrate middleware-services (security, transaction processing, naming, persistence,..),

- to deliver an application independent user-interface to allow specialized extensions.

A framework is a good approach to serve these requirements. The framework should consist of bricks for the user interaction (workflow development and runtime support and representation of all business objects) and the integration of existing applications, decentralized control, integration of open platforms including middleware. Thus, a WfMS can be composed effectively of parts of the framework.

The paper describes, in short, the design rules of BPAFrame (Framework for Business Process Automation) and its main components, and especially the components for the communication with the users. Our current prototype implementation (for environmental information systems) is presented, together with an example application. And finally we make a reference to future areas of improvement in the conclusion.

\section{DESIGN OF BPAFRAME}

Main requirements for a new and basic approach to WfMS are as follows:

- modularization: is supported by a framework, a set of components;

- individualization: is supported by easy integration of business data and workflows;

- scaleability: means effective solutions a whole range of application sizes;

- modification: demands easy and fast reaction to changes in the organization and in the tasks;

- comprehensibility: requires protocols of workflow events and referenced data;

- availability: is based on failure independence, reliable control mechanisms and trustworthy feedback; 
- reuse of existing application software: allows a transparent management of user data, but needs interfaces to OLE, OpenDoc,...;

- platform independence: is supported by the use of a GUI-independent software development tool.

To meet these requirements our approach demands modularization within a framework and generic applications. All distribution aspects shall be hidden by the transparent use of an open platform for distributed object oriented applications

\subsection{Basic rules of BPAFrame}

BPAFrame is designed and implemented as an agent based framework for business process automation. The core of the system is the decentralized control represented by the processing model (see figure 1). It consists of three classes (Task, Task-Description and Agent).

The business task description (workflow type) represents exactly the agent's steps during the processing of a task in an organization (enterprise,.. department) and declares the required parameters. The business task is based on the task description. Additionally it contains the owner of the task, current data, etc. The agent is an expansion of the object-oriented paradigm, it realizes active objects (without own intelligence). The agent processes a business task step-by-step (i.e. one agent per task). It controls the workflow interpreting the task description (represented in AgentScript, an effective interpretable, internal textual description of business tasks). It saves the output parameters and other protocol data, too.

Sub-workflows are realized by the instantiation of new agents with their own sub-scripts. Parallelity is realized by the creation of independent agents.

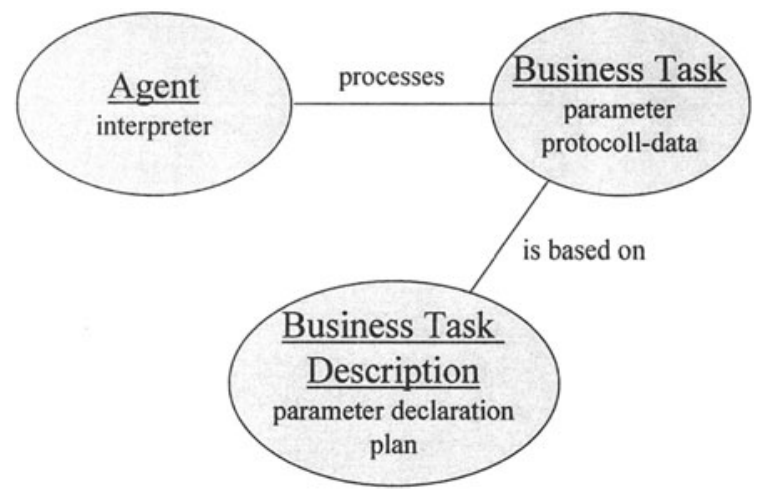

Figure 1: Basic components of BPAFrame

As mentioned above, Workflow-Management-Systems have to work in a distributed fashion, as they are using the networked infrastructure of the organization. The use of an open middleware platform allows the integration of a wide range of computer hardware. Thus, we used an CORBA- implementation (Common Object Request Broker Architecture) as the distribution platform. The ORB (Object Request Broker) invokes methods independent of 
their location and independent of their implementation details. Moreover, methods can be invoked dynamically (their location may be unknown at the compilation-time of the application).

The ORB implementation should be compatible with the standard CORBA 2.0 (Common Object Request Broker Architecture (OMG, 1995 (1)) of the Object Management Group) and should additionally contain Object Services (OMG, 1995 (2)) (CORBAservices security, persistence,..). CORBA 2.0 defines the interoperability between ORB-implementations. Therefore, it allows interaction in a simple manner with objects managed by other ORBimplementations. And furthermore, it makes the cooperation of BPAFrame with other CORBA-based WfMS possible. Thus, the system is able to communicate with OLE-objects (as MS-Word or MS-Excel documents), too. Most ORBs already contain a linkingfunctionality to OLE-Objects.

The design-process of the framework for WfMS results in an easy to handle, general purpose model of the business objects of an organization. Therefore, it can be used for more effective task processing as well as for better improving organizational structures.

\subsection{The architecture of BPAFrame}

BPAFrame consists of a set of modules, as shown in figure 2. It contains development and runtime support and user interaction services for workflows in open distributed systems.

The business process framework realizes the decentralized control. Thus, it fulfills the demands of chapter 2.1: It uses the agent framework with active objects and all necessary runtime support (e.g. an interpreter for AgentScript - the internal workflow representation language). Together with the organization structure library the user services framework uses the business process framework for all purposes of initialization, supervision and storing of workflows and the active participation of users within workflows

The rate of acceptance for WfMS depends mainly on two factors:

- "look and feel" and user-support for all, the development of workflows, the implementation of the organizational structure and the runtime support of the employees, respectively and

- sufficient security integration for all the application needs (range of access rights).

That's why, the services for the user interaction are one of the focal points of the development of BPAFrame. These services contain its own framework within the BPAFrame, called the User Services Framework. 


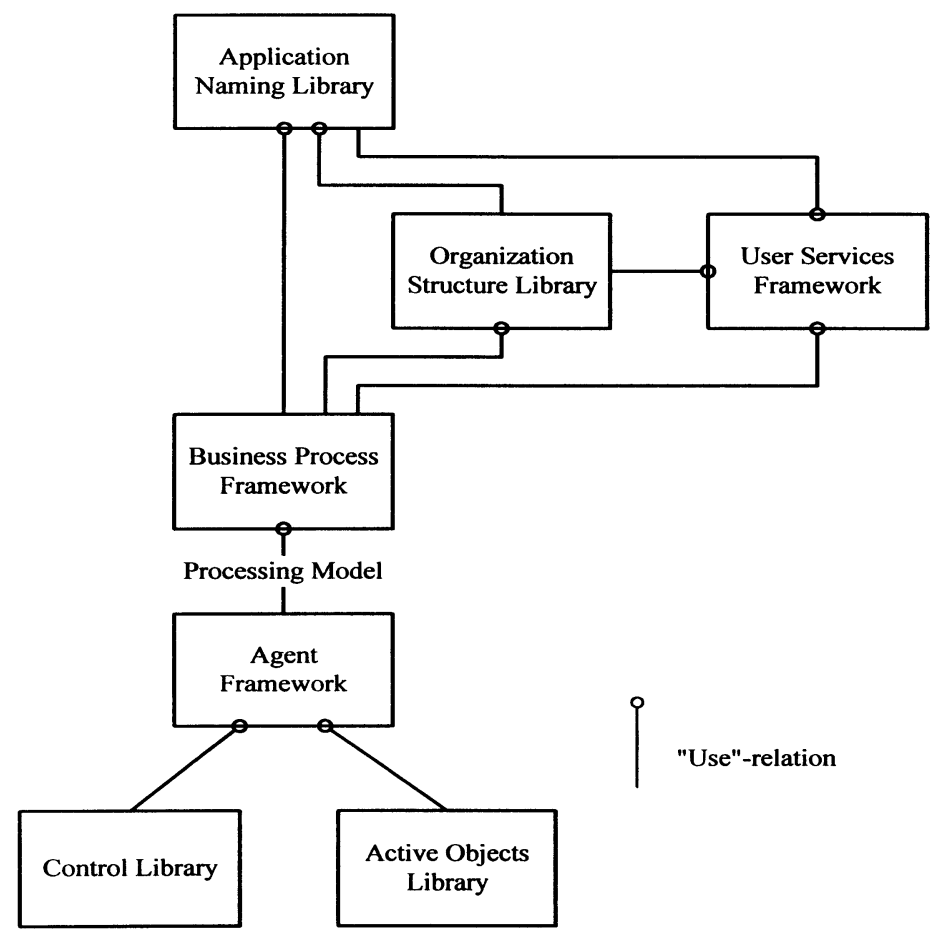

Figure 2: Modules of BPAFrame

\subsection{The user services framework}

The User Services Framework contains a set of services for user communication. Two important aspects of user communication have to be discussed:

First, interaction servers are required for the interaction between the users and their software representation in BPAFrame. The representatives of users in BPAFrame are specialized agents called "user agents". User agents are always aware of the employee's state (login/logout, and on which computer);

Second, a workspace has to be built representing the front-end application for the user.

The user services contain the workspace functions for the users and the task and interaction management (see figure 3). Thus, it is always able to correspond with the WfMS, even if the users is not presently logged in. A queuing mechanism handles the incoming tasks, which the user has to do, which implies that the user interactions are serializes too. 


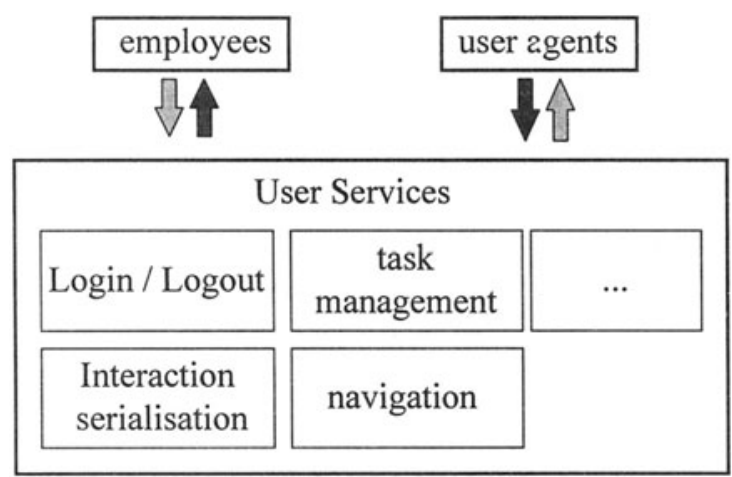

Figure 3: User Services for the interaction management between employee and user agent

Additionally, the workspace has to consider the following tasks for the user interactions, and has to manage the cooperation of the WfMS and other locally running applications:

- authenticate users;

- initiate tasks, which the user want to have executed from the organization (including authorization);

- inform the user about the state and the termination of initiated tasks;

- abort initiated tasks;

- show tasks, which its employees must work on (i.e. to give him an overview and to allow him the selection).

Thus, the user server is designed to deliver these basic mechanisms in the modules of figure 3. Consequently there is no restriction neither in the platform the workspace runs nor in the combination of functions that the front-end contains. So, you can program your own workspace, your own front-end for the WfMS based on BPAFrame very easily and quickly with this user services framework. And, it is easy to develop front-ends for different operating systems, only this framework contains GUI-dependent code.

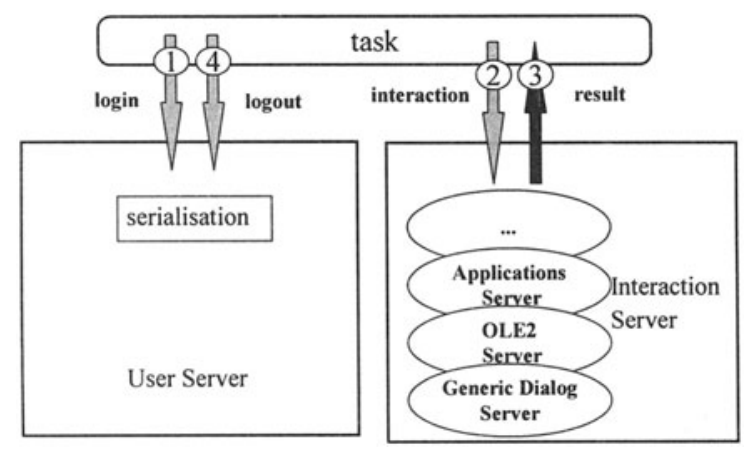

Figure 4: Interaction protocol of all user services 
The user agent interacts with the user, if a task is selected by the user. Then, the interaction protocol of figure 4 is always used. The user server tests the authentication of the user (and the authorization rights of the task) (1). After which (2) the suitable interaction server is selected. After finishing the application, the results are reported back to the task (to the workflow agent) (3) and the interaction terminates (4). The workflow agent then follows the next step(s) of its workflow script processing the workflow plan.

The user service framework also contains interaction servers for the communication with different types of application software, see fig. 4. The interaction server has the responsibility for the necessary communication between the user agent (representing the current task) and the user during the processing of a task. Interaction servers exist for different types of applications, for instance application servers, generic dialogue servers and OLE2-servers. There is the possibility to enlarge the system by using/adding new interaction servers, e.g. for OpenDoc. And, there is no need for re-compilation of the whole workflow-managementsystem if the workspace is extended. Simply the new interaction server has to be invoked in the script of the task description (AgentScript). No more changes are necessary for the integration of such a new server.

The generic dialogue servers are important interaction servers for the communication with the user. Different generic dialogue servers should exist for each operating system working in the heterogeneous system. In order to become independent from the operating system, there must exists the same programming interface for each dialogue server. The input parameters of the generic dialogue server define the appearance of the dialogue window and the output parameters. Thus, it is easy to develop a specific dialogue very quickly.

Therefore, a workspace contains at least such a generic dialogue server, as well as an application server invoking the legacy application.

\section{PROTOTYPE AND APPLICATION SCENARIO}

Our current implementation of the BPAFrame runs as a prototype for evaluation purposes. It uses Windows NT 3.5 and the Object-Request-Broker ORBeline 1.0 (PostModernComputing, 1995). The goal of the implementation of the system has been the evaluation of the processing model and the user communication. We have programmed a workspace, where we can initiate tasks and interaction servers and interact with the user.

In our prototype we are using workflows of daily tasks in environmental departments of districts and cities. They have to evaluate, for instance, contaminated areas, development plans, building applications, etc. Different employees have to take part in such evaluation processes, they have to access databases (situated in different locations), to start simulations, to analyze and to integrate test results and they have to make decisions. Thus, WfMS meet these requirements well. Furthermore, they allow improved (i.e. faster and more reliable) processing of the departments workflows. BPAFrame is able to control all of the required parallel sub-workflows, e.g. the evaluation of water pollution, ground water pollution, etc. 


\section{CONCLUSION}

Our next steps in the development of the BPAFrame will be focussed on improving the following components: integration of better security services; testing of different ORBimplementations and a better interaction service for the development of workflows. BPAFrame Phase 2 will use Orbix (IONA, 1995) representing a reliable and fast CORBAimplementation.

Thus, we have to observe the development of the CORBA-standards of the OMG. And, we are testing different object request broker implementations as well. One of the most interesting features of CORBA is the interoperability between CORBA implementations. Its application for BPAFrame is one of our most challenging tests in the near future. That will help to successfully simplify the communication between applications on top of different operating systems and computer architectures.

\section{REFERENCES}

IONA Techn.Ltd. (1995) Orbix 2 - Distributed Object Technologie, Programmer's Guide, Dublin

Joosten, S.and Brinkkemper, S. (1995) Fundamental Concepts for Workflow Automation in Practice. ICIS `95, Amsterdam

Mittasch, Ch., Funke, R. and König, W. (1996) Trader supported Distributed Office Applications in IFIP/IEEE Int. Conf. on Distributed Platforms, Dresden

Mittasch, Ch., Irmscher, K. (1994) On the Way to Competitive Market of Services in Heterogeneous Networks in Conf. Vol. 2 (ed. Raubold) of IFIP World Computer Congress, 57-64

Mittasch, Ch. and Schill A. (1996) CodAlf: A Decentralized Workflow Management System on Top of OSF DCE and DC++ submitted paper to ACM 1996 Conference on CSCW, Boston, November 1996

OMG (1995) (1) The Common Object Request Broker: Architecture and Spec., Rev. 2.0

OMG (1995) (2) CORBAservices: Common Object Services Specification. Revised Edition 1995

PostModernComputing (1995) Orbeline Documentation, Version 1.0

Schill, A. and Mittasch Ch. (1996) Workflow Management Systems of Top of OSF DCE and OMG Corba, submitted for publication in Distributed Systems Engineering Journal, September 1996 